\title{
EL CONCEPTO DE LUCHA EN LA SOCIOLOGIA DE BOURDIEU
}

\section{THE CONCEPT OF STRUGGLE IN BOURDIEU'S SOCIOLOGY}

\author{
Julieta María Capdevielle* \\ María Laura Freyre**
}

RESUMEN

Es posible leer la obra de Bourdieu como una búsqueda por explicar y comprender los mecanismos a través de los cuáles se perpetúa el orden social desigual. Sin embargo, no se puede desconocer que la obra de Bourdieu nunca consideró incompatible la descripción de tendencias reproductivistas con la investigación de tendencias contrarias. Así, este artículo intenta aportar en el análisis de la distinción entre la lucha de clases en su forma competitiva y la lucha de clases en sentido revolucionario para la transformación social.

PALABRAS CLAVE: BOURDIEU * LUCHA * CLASES SOCIALES * PODER * VIOLENCIA SIMBÓLICA

\section{ABSTRACT}

You can read the work of Bourdieu as a quest to explain and understand the mechanisms through which perpetuates the unequal social order. However, there is no denying that the work of Bourdieu never considered incompatible reproductivists description of the research trends of contrary tendencies. Thus, this article tries to contribute in the analysis of the distinction between the class struggle in a competitive and class struggle in a revolutionary sense for social transformation.

KEYWORDS: BOURDIEU * STRUGGLE * SOCIAL CLASSES * POWER * SYMBOLIC VIOLENCE

Centro de Investigaciones de la Facultad de Filosofía y Humanidades (CIFFYH) "María Saleme de Burnichón" de la Universidad Nacional de Córdoba (UNC), Argentina.

laufreyre@gmail.com

** Centro de Investigaciones de la Facultad de Filosofía y Humanidades (CIFFYH) "María Saleme de Burnichón" de la Universidad Nacional de Córdoba (UNC), Argentina.

julietacapdevielle@gmail.com 


\section{INTRODUCCIÓN}

En el estructuralismo genético ${ }^{1}$ de Pierre Bourdieu, el poder es presencia ineludible y este aparece como relación, como relación de fuerzas, como enfrentamiento y lucha. Así, se define a la sociedad como un espacio diferenciado de posiciones, que no forma una totalidad única integrada por funciones sistemáticas y una única cultura común. Por el contrario, se reconoce la diferencia, la desigualdad y la lucha por el control de los recursos individuales y sociales (Cragnolino, 2011: 5). El análisis hace foco en el trazado de los movimientos, estrategias y apuestas de los distintos agentes dentro del espacio social, al cual le confiere un carácter dinámico, una relación entre intereses confrontados que encuentran su síntesis y fuerza en el concepto de "lucha". Dicho concepto enriquecido con la incorporación de las dimensiones culturales, simbólicas, morales, psicológicas y corporales.

La lucha de clases se vuelve, desde la perspectiva de Bourdieu, cotidiana y se juega en las escenas más triviales de la vida diaria. Pero, a su vez, la potencialidad del concepto de lucha reside, desde nuestro punto de vista, en que este se alza como la bisagra sobre la cual se asienta la posibilidad o no de la transformación del orden social. En síntesis, el concepto de lucha adquiere un lugar clave en la perspectiva

1 "Por estructuralismo o estructuralista, quiero decir que existen en el mundo social, y no solamente en los sistemas simbólicos, lenguaje, mito, etc., estructuras objetivas, independientemente de la conciencia y de la voluntad de los agentes, que son capaces de orientar o de coaccionar sus prácticas o sus representaciones. Por constructivismo, quiero decir que hay una génesis social de una parte de los esquemas de percepción, pensamiento y acción que son constitutivos de lo que llamo habitus, $y$ por otra parte estructuras, $y$ en particular de lo que llamo campos y grupos, especialmente de lo que se llama generalmente clases sociales" (Bourdieu, 2007a: 127). Así, "basado en una ontología social no cartesiana que rechaza la división entre objeto y sujeto, intención y causa, materialidad y representación simbólica, Bourdieu busca superar la reducción de la sociología ya sea a una física objetivista de las estructuras material o a una fenomenología constructivista de las formas cognitivas, mediante un 'estructuralismo genético' capaz de incluir a ambas” (Bourdieu y Wacquant, 2008: 28). bourdieussiana; sin embargo, ha sido poco abordado por los cientistas sociales. Con el presente artículo, se pretende aportar en dicho sentido, dando cuenta de las distintas modalidades y dimensiones que adquiere el mencionado concepto en la obra del sociólogo francés.

Para este análisis, se ha indagado principalmente en sus obras La Distinción (2000); Sociología y Cultura (1990); ¿Qué significa hablar? Economía de los intercambios lingüísticos (2001); en las entrevistas compiladas en Cosas dichas (2007e) y en Respuestas para una antropología reflexiva (Bourdieu y Wacquant, 2008). En esos textos, creemos encontrar sus elaboraciones más importantes en torno a las modalidades $y$ dimensiones que adquiere el concepto de lucha en su teoría.

\section{LA SOCIEDAD COMO ESPACIO SOCIAL DE POSICIONES}

Desde el marco de la perspectiva estructuralista que integra el modo de pensamiento relacional con la incorporación de la dimensión histórica, Pierre Bourdieu concibió al espacio social como un espacio que define acercamientos y distancias sociales; en él no se pueden ignorar las diferencias objetivas fundamentales. Así, el espacio social se caracteriza por la existencia de diferencias sociales, por el acento en la desigualdad ligada a la distribución de recursos eficientes (los capitales) y con ello, la cuestión del poder como constitutivo de la sociedad (Cragnolino, 2011: 7).

La característica distintiva del espacio social bourdieussiano ${ }^{2}$, tal como se analiza hasta el momento, es que en él los agentes luchan, según sus posiciones ${ }^{3}$, ya sea para cambiar o

$2 \quad$ El espacio social bourdieussiano se diferencia del espacio geográfico, aunque ambos espacios se relacionan, ya que el espacio geográfico, trasluce las diferencias y distancias de las posiciones ocupadas en el espacio social. De este modo, la apropiación del espacio geográfico, depende del lugar ocupado en el espacio social. Para mayores referencias sobre este tema ver Efectos de Lugar (Bourdieu, 2007d: 119-124).

3 Posiciones que, desde esta perspectiva, son independientes de la voluntad y de la conciencia porque están estructuralmente determinadas. 
para preservar las fronteras o las formas de dicho espacio; en definitiva, las reglas de división y transformación de las posiciones de los agentes en el espacio social (Bourdieu y Wacquant, 2008: 42). Dicha lucha se asienta siempre en un "sistema" bidimensional de relaciones de poder: por un lado, relaciones objetivas (lucha por determinados recursos) y por otro, relaciones de significado entre grupos y clases (lucha por imponer una visión legítima sobre el mundo social). De este modo, si bien Bourdieu, conjuntamente con Marx, veía en las relaciones sociales fuerzas objetivas, también puso en el centro de su interrogación sociológica las relaciones de sentido, aspecto que tomó de la obra de Max Weber.

Desde esta mirada, la sociología como ciencia crítica, debe realizar una doble lectura de lo social, donde se aborde el "sistema" de relaciones de poder y las relaciones de significado entre grupos y clases. Así, la primera aproximación concibe a la sociedad en la manera de una física social: como una estructura objetiva, constituida por la "distribución" de recursos "materiales" y medios de apropiación de bienes y valores socialmente escasos (especies de capital), captada desde afuera, cuyas articulaciones pueden ser materialmente observadas y mesuradas, independientemente de las representaciones que se hagan aquellos que en ella viven. La fuerza de este punto de vista objetivista o "estructuralista" reside en socavar la "ilusión de la transparencia del mundo social" (Bourdieu y Wacquant, 2008: 31). Desde este posicionamiento teórico, el sentido de las acciones en principio más personales y más "transparentes" no pertenece al sujeto que las lleva a cabo, sino al sistema total de relaciones en las cuales y por las cuales, se realizan. Es decir, las relaciones sociales no pueden reducirse a relaciones entre subjetividades animadas de intenciones o "motivaciones", porque ellas se establecen entre condiciones $y$ posiciones sociales y tienen, al mismo tiempo, más realidad que los sujetos que relacionan ${ }^{4}$ (Bourdieu, Chamboredon y Passeron, 2008: 37).

4 Esta forma de concebir la acción social es la que diferencia la perspectiva bourdieussiana de otras posturas sociológicas como la Teoría de la Elección Racional y del Interaccionismo Simbólico.
Sin duda, la sociedad tiene una estructura objetiva, pero no es menos cierto que también se compone de modo decisivo de "representaciones y de voluntad" (Bourdieu y Wacquant, 2008: 32). De este modo, la segunda lectura busca captar la forma de los sistemas de "clasificación", esquemas mentales y corporales que funcionan a manera de patrones "simbólicos" para la actividad de las prácticas, conductas, pensamientos, sentimientos y juicios de los agentes sociales. Más cercano a un inconsciente de clase que a una "conciencia de clase" en el sentido marxista, el sentido de la posición ocupada en el espacio social es el dominio práctico de la estructura social en su conjunto, que se ofrece mediante el sentido de la posición ocupada en esa estructura (Bourdieu, 1990: 289) y es este sense of ones's place o sentido de la posición, parte importante y constituyente del habitus de los agentes sociales. Las categorías de percepción del mundo social son, en lo esencial, el producto de la incorporación de las estructuras objetivas del espacio social. En consecuencia, inclinan a los agentes a tomar el mundo tal cual es, a aceptarlo como natural más que a rebelarse contra él, a oponerle mundos posibles, diferentes y aún, antagonistas (Bourdieu, 1990: 289). Es en este sentido, que las relaciones de fuerza están también presentes en las conciencias, bajo la forma de categorías de percepción de esas relaciones. En esta instancia subjetivista, es cuando la sociología busca dar cuenta del sentido que los agentes le otorgan a sus prácticas y al mundo social, lo que también forma parte de la realidad bajo estudio.

Por lo tanto, la sociología debe evitar, desde esta mirada teórica, dos reduccionismos: el objetivista y el subjetivista. En otras palabras, la sociología debe "superar la oposición" entre una "física social" que, armada con un uso objetivista de la estadística, establecería unas distribuciones (en el sentido estadístico y también en el económico) y una "semiología social" que se aplicaría a descifrar unas significaciones $y$ a poner de manifiesto las operaciones cognitivas mediante las cuales los agentes las producen y las descifran (Bourdieu, 2000: 493). Es decir, el mundo social para la teoría sociológica de Bourdieu, no solo se compone de estructuras 
objetivas, sino también de representaciones, percepciones $y$ visiones. Los sistemas simbólicos contribuyen a construir al mundo y dotarlo de sentido para quienes viven en él. La lucha en el espacio social y en los distintos campos $^{5}$ posee esta doble dimensión: "simbólica" y "material". Se retomará el análisis de estas dimensiones con mayor profundidad a lo largo del artículo.

\section{CLASES, LUCHA DE CLASES, LUCHA POR LAS CLASIFICACIONES}

\section{LA TEORÍA DE LAS CLASES SOCIALES BOUR- DIEUSSIANA}

Tal como se analizó, la definición del espacio social bourdieussiano se funda en una concepción agonística de lo social, sobre la que se asienta el concepto de "lucha". A partir de los años 1960 y sobre todo después de su obra La distinción, Bourdieu elaboró una concepción mucho más compleja de las relaciones de clases. Así, en la construcción de su teoría de las clases sociales, realiza una serie de rupturas con la teoría marxista. En primer lugar, se distancia de cierto marxismo por su tendencia a privilegiar las sustancias en detrimento de las

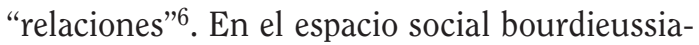
no, las posiciones y las tomas de posiciones de los agentes se definen siempre relacionalmente.

$5 \quad$ En términos analíticos, un campo puede ser definido como una red o una configuración de relaciones objetivas entre posiciones. Estas posiciones están objetivamente definidas, en su existencia $y$ en las determinaciones que imponen sobre sus ocupantes, agentes o instituciones, por su situación presente y potencial en la estructura de distribución de especies de poder (o capital) cuya posesión ordena el acceso a ventajas específicas que están en juego en el campo, así como por su relación objetiva entre posiciones (dominación, subordinación, homología, etc.) (Bourdieu y Wacquant, 2008: 134-135).

Para Bourdieu, la tendencia a privilegiar las sustancias en detrimento de las "relaciones" en la teoría marxista lleva a confundir la clase teórica o "clase en el papel", construida científicamente, con una clase real, un grupo efectivamente movilizado (Bourdieu, 1990: 281).
En segundo lugar, esta teoría de las clases critica la visión marxista del concepto de capital, al referirlo a lo estrictamente económico como una forma de reduccionismo. En la sociología de Bourdieu, el control diferencial de recursos críticos - capitales-constituye el principio de definición de posiciones e intereses antagónicos, desde donde se hacen comprensibles y explicables las prácticas. Estos recursos desde esta perspectiva sociológica, no se reducen a lo económico, sino que son los que en cada sociedad $y$ en diferentes momentos históricos de la misma, se han constituidos como tales, por el hecho de reunir dos características fundamentales: ser escasos y al mismo tiempo, valiosos. En la medida en que un recurso escaso sea objeto de interés por parte de diversos actores, el control diferenciado del mismo (por propiedad, posesión o administración) dará lugar a que algunos agentes sociales se encuentren en posición de imponer condiciones a quienes quieran acceder a él y esta situación implica la instalación de las relaciones básicas de dominación identificadas por Marx desde la propiedad de los medios de producción (Costa, 2006: 169-170).

Por último, en la teoría de las clases sociales bourdieussiana, se encuentra la ruptura con el objetivismo marxista que lleva a ignorar las luchas simbólicas y su disputa por la representación misma del mundo social. De esta manera, una clase social no solo posee propiedades ligadas a relaciones objetivas que mantiene con las demás clases, sino también posee propiedades ligadas a relaciones simbólicas. Se trata de distinciones significantes, que tienden a reduplicar simbólicamente las diferencias de clases en el plano objetivo. Los sistemas de clasificación constituyen un asunto que está en juego en las luchas que oponen a individuos o grupos en las interacciones rutinarias de la vida diaria, así como también en las contiendas solitarias y colectivas que tienen lugar en los campos de la política y de la producción cultural (Bourdieu y Wacquant, 2008: 39). Las clases y otros colectivos sociales antagónicos están continuamente comprometidos en una lucha por imponer la definición del mundo que resulta más congruente con sus intereses particulares (Ibid., 2008: 39). 
Con ello, Bourdieu propone la construcción de un "espacio social" como una estructura de posiciones diferenciadas, en donde las clases sociales no se definen por una propiedad ni por la suma de las propiedades (propiedades de sexo, de edad, de origen social o étnico), sino por la estructura de las relaciones entre todas las propiedades pertinentes, que confiere su propio valor a cada una de ellas y a los efectos que esta ejerce sobre las prácticas.

La configuración singular del sistema de los factores explicativos que hace falta construir para explicar un estado de distribución de una clase particular de bienes o de prácticas, es decir, de un balance, confeccionado en un momento dado del tiempo, de 'la lucha de clases' que tiene como apuesta esa categoría particular de bienes o de prácticas (caviar o pintura de vanguardia[...]) es la forma que toma, en este campo, el capital objetivado (propiedades) e incorporado (habitus) que define en propiedad la clase social y que constituye el principio de producción de prácticas distintivas, es decir, enclasadas y enclasantes; representa un estado del sistema de propiedades que hacen de la clase un principio de explicación y de clasificación universal, que define el rango ocupado en todos los campos posibles (Bourdieu, 2000: 112-113).

Siguiendo las palabras de Bourdieu, las clases sociales son construidas como el conjunto de agentes que ocupan posiciones semejantes $y$ que situados en condicionamientos semejantes y sometidos a condicionamientos semejantes, tienen todas las probabilidades de tener disposiciones e intereses similares $y$ de producir, por lo tanto, prácticas y tomas de posiciones semejantes (Bourdieu, 1990: 284).

\section{LAS DIMENSIONES DE LA LUCHA DE CLASES}

Tal como se comenzó a esbozar a lo largo de este artículo, Bourdieu retoma los aportes de Marx y Weber para analizar el espacio social como un espacio de lucha, como sistema de relaciones objetivas y simbólicas en el que las posiciones y la toma de posiciones se definen relacionalmente. En este espacio - que incorpora la dimensión histórica y relacional- pueden comprenderse las estrategias individuales o colectivas, espontáneas u organizadas, las cuales tienen como punto de vista el conservar, el transformar o el transformar para conservar, las relaciones de fuerza que sustentan las distancias sociales en el espacio y la desigualdad social (Bourdieu, 2000: 156). De este modo, las luchas fijan un estado de la distribución de los distintos capitales que determinan las ventajas $y$ obligaciones de los agentes. El control diferencial de los distintos recursos otorga solo a algunos, la capacidad de imponer condiciones a quienes quieran acceder a estos.

Así, Bourdieu incorporó en el análisis de las relaciones de clase una multiplicidad de dimensiones - culturales, simbólicas, morales, psicológicas y corporales- $y$ a su vez, reintrodujo a los individuos $y$ a la vida cotidiana en los análisis de clase. La lucha de clases se vuelve desde esta perspectiva algo cotidiano y se desarrolla en las escenas más triviales de la vida diaria.

Una de los aspectos centrales de las disputas en la teoría bourdieussiana $-y$ en ruptura con la visión marxista de las clases sociales - lo constituye la dimensión simbólica. Dimensión a la que se abocará con mayor detenimiento en el siguiente apartado.

\subsection{LA LUCHA SIMBÓLICA COMO ACTO DE CONSTRUCCIÓN DEL MUNDO SOCIAL}

La lucha simbólica por la producción del sentido común o más precisamente, por la visión legítima del mundo social, constituye en la teoría de Bourdieu, parte central de la lucha de clases. Así, la lucha implica, a su vez, relaciones de poder que adquieren una dimensión simbólica, en tanto que lo que se disputa en ellas es la representación misma del mundo social. Con ello, Bourdieu recupera la autonomía relativa de la lógica de las representaciones simbólicas en relación a los determinantes materiales ${ }^{7}$. Esas

$7 \quad$ Basta con tener presente que los esquemas clasificadores que se encuentran en la base de la relación práctica que mantienen los agentes con su condición y que la representación que pueden tener de ellos, son a su vez, producto de esa condición, para ver los límites de la autonomía: la posición en la lucha de enclasamientos depende de la posición en la estructura de las clases (Bourdieu, 2000: 494). 
representaciones, por medio de su propia eficacia, pueden ayudar a perpetuar o a subvertir dichas relaciones de poder.

"De hecho, el trabajo de categorización, es decir, de explicitación y de clasificación, se realiza sin cesar, en todo momento de la vida diaria" (Bourdieu, 1990: 290). Por lo cual, la lucha de clases no constituye solo el principio mismo de distribución de los bienes escasos, sino que también, en ella se juega el modo legítimo de percibirlos. De este modo, a través de las luchas, los agentes disputan tanto el sentido del mundo social como su posición en ese mundo, en definitiva, lo que está en juego es la identidad social de quienes participan en la disputa.

Cabe aclarar que la representación del mundo social no es fija, sino por el contrario, es producto de innumerables acciones de "construcción", siempre ya hechas y siempre por re- hacer (Bourdieu, 2007c: 171). En parte esto se debe a que los "objetos del mundo social" se pueden percibir y decir de diferentes maneras porque, como los objetos del mundo natural, comportan siempre una parte de "indeterminación" y "evanescencia" que se debe a que aún las combinaciones más constantes de propiedades (...) están sometidas a variaciones de orden temporal y a que su propia significación, en la medida en que está suspendida en el futuro, está en suspenso, en espera y por lo tanto, relativamente indeterminada (Bourdieu, 1990: 288). Esta parte de juego y de incertidumbre, es la que da fundamento a la pluralidad de las visiones del mundo, asimismo, está vinculada con la pluralidad de puntos de vista, con todas las "luchas simbólicas” por la producción e imposición de la visión de mundo legítima y más precisamente, con todas las estrategias cognitivas de "llenado" que producen el sentido de los objetos del mundo social más allá de los atributos directamente visibles por la referencia al futuro o al pasado (Bourdieu, 1990: 288).

Recodar que la percepción del mundo social entraña un acto de construcción, no implica en modo alguno aceptar una teoría intelectualista del conocimiento: lo esencial de la experiencia del mundo social y del trabajo de construcción que esta experiencia implica se opera en la práctica, sin alcanzar el nivel de la representación explícita ni de la expresión ver$\mathrm{bal}^{8}$ (Bourdieu, 1990: 288-289).

Para sostener este argumento, a modo de ejemplo, se cita las palabras de Raymond Williams, quien recuerda que la percepción del propio mundo natural no tiene nada de natural, en particular, gracias a la verdadera genealogía social de las categorías de percepción del mundo natural y también, porque ella es indisociable de una relación con el mundo social. El punto de vista sobre el mundo natural y a fortiori, sobre el mundo social, dependen de la altura social desde donde se tome (Bourdieu, 2007c: 174).

8 De este modo, en la teoría bourdieussiana, las prácticas son el producto del aprendizaje del juego social; que le permite saber al agente lo que hay que hacer en una situación determinada - lo que en el deporte se llama el sentido del juego-, arte de anticipar el desarrollo futuro del juego. Así, la noción de estrategia en la teoría de Bourdieu cobra relevancia, en tanto es el producto del sentido práctico como sentido del juego incorporado por los agentes. Un juego social particular, históricamente definido, que se adquiere desde la infancia al participar en las actividades sociales. El buen jugador, dice Bourdieu, es en cierto modo el juego hecho hombre, hacer en cada instante lo que hay que hacer, lo que demanda y exige el juego. Desde la noción de estrategia y habitus, Bourdieu critica la concepción de sujeto de la teoría de la acción racional. Esta no reconoce nada salvo "respuestas racionales" a oportunidades potenciales o realidades de un agente que es tan indeterminado como intercambiable. Para Pierre Bourdieu, la teoría de la acción racional peca al ignorar tanto los condicionamientos sociales como la historia individual y colectiva de los agentes. Tomando distancia de esta postura, Bourdieu propone una teoría de la acción (con la noción de habitus) donde la mayor parte de las acciones humanas tienen como principio algo absolutamente distinto de la intención, es decir, disposiciones adquiridas que hacen que la acción pueda $y$ tenga que ser interpretada como orientada hacia tal o cual fin, sin que pueda plantear por ello, que como principio tenía el propósito consciente de ese fin (Bourdieu, 1999b: 166). Es decir, el habitus en la teoría de Bourdieu no deja de ser pre-reflexivo, lo que hace que el agente social no se comporte como el sujeto calculador de la economía racional. 
En resumen: "la construcción social de la realidad social" se realiza en y por innumerables actos de construcción antagonistas que los agentes operan, en cada momento, en su luchas individuales o colectivas para imponer la representación del mundo social más favorable a sus intereses; lucha ciertamente muy desigual, ya que los agentes tienen un dominio muy variable de los instrumentos de producción de la representación del mundo social (Bourdieu, 2007c: 171). De este modo, la fuerza de que disponen los agentes depende también (además del volumen y estructura del capital ${ }^{9}$ que poseen), del "estado de la lucha con respecto a la definición de la apuesta de la lucha". Se trata de una lucha simbólica por la definición de los instrumentos y de las apuestas legítimas, y por el porcentaje de conversión de las distintas especies de capital.

$9 \quad$ No se puede dejar de mencionar la importancia del "capital simbólico", como un recurso fundamental en las luchas por la construcción social de la realidad y por imponer la representación del mundo social. El capital simbólico constituye uno de los instrumentos que intervienen de modo central en la construcción de la representación del mundo social y es uno de los recursos que se haya mas desigualmente distribuido. El capital simbólico - otro nombre de distinción- no es sino el capital, de cualquier especie, cuando es percibido por un agente dotado de categorías de percepción que provienen de la incorporación de la estructura de su distribución, es decir, cuando es conocido y reconocido como natural. Las distinciones, en su calidad de transfiguraciones simbólicas de las diferencia de hecho y más en general, los rangos, órdenes, grados o todas las otras jerarquías simbólicas, son el producto de la aplicación de esquemas de construcción que, como por ejemplo, los pares de adjetivos empleados para enunciar la mayoría de las valoraciones sociales, son el producto de la incorporación de las estructuras a las que se aplican y el reconocimiento de la legitimidad más absoluta no es sino la aprehensión como natural del mundo ordinario que resulta de la coincidencia casi perfecta de las estructuras objetivas con las estructuras incorporadas (Bourdieu, 1990: 293). De ello se concluye, entre otras consecuencias, que el "capital simbólico" y que la autonomía real del campo de producción simbólica no impide que este siga dominado, en su funcionamiento, por las fuerzas que rigen el campo social, ni que las relaciones de fuerza objetivas tiendan a reproducirse en las relaciones de fuerza simbólicas, en las visiones del mundo social que contribuyen a asegurar la permanencia de las relaciones de fuerza (Bourdieu, 1990: 293).
Aquella es una de las apuestas fundamentales de las luchas (y por lo tanto, está expuesta a cambios incesantes) entre las distintas fracciones de clase, cuyos poderes y privilegios están vinculados a una u otra de esas especies de capital (Gutiérrez, 2007a: 20-21).

\section{MODALIDADES DE LUCHA}

\section{LUCHA COMPETITIVA Y LUCHA POLÍTICA: CON- SERVACIÓN VS REVOLUCIÓN}

Se encuentra en la teoría bourdieussiana que las luchas sociales no están todas ni siempre en contradicción con el orden establecido. Así, es posible distinguir en su obra dos grandes modalidades de lucha: la "lucha competitiva" y la "lucha revolucionaria". La primera, la lucha competitiva, se caracteriza en su funcionamiento por asegurar la reproducción del orden social y con ello, la reproducción de las relaciones de dominación/dependencia. De este modo, esta modalidad de lucha no amenaza necesaria ni automáticamente, la supervivencia del sistema. Así, la reproducción de la estructura social puede realizarse dentro y por medio de la lucha competitiva que conduce a una simple traslación de la estructura y con ello, a una traslación y perpetuación de las diferencias iniciales.

Esta forma particular de lucha de clase que es la lucha competitiva es la que los miembros de las clases dominadas se dejan imponer cuando aceptan las apuestas que les proponen los dominantes, lucha integradora y, a causa de su handicap inicial, reproductora, puesto que los que entran en esta especie de carrera-persecución en la que parten necesariamente derrotados, como testimonia la constancia de las diferencias, reconocen implícitamente, por el solo hecho de competir, la legitimidad de los fines perseguidos por aquellos a quienes persiguen (Bourdieu, 2000: 164-165).

La lucha competitiva se caracteriza así, por ser una lucha individual $y$ aislada, que condena a los agentes a actuar ante el efecto de las innumerables reacciones de los grupos de agentes. 
Un proceso semejante de desarrollo homotético se observa, según parece, siempre que las fuerzas y los esfuerzos de los grupos en competencia por una especie determinada de bienes o de titulaciones especiales tienden a equilibrarse como una carrera en la que, al término de una serie de adelantamientos $y$ recuperaciones, se mantienen las diferencias iniciales, es decir, siempre que las tentativas de los grupos inicialmente más carentes por apropiarse los bienes y las titulaciones poseídos hasta entonces por grupos situados inmediatamente por encima de ellos en la jerarquía social, o inmediatamente delante de ellos en la carrera, resulta casi compensadas, en todos los niveles, por los esfuerzos que hacen los grupos mejor situados por mantener la singularidad $y$ la distinción de sus bienes y de sus titulaciones (Bourdieu, 2000: 160).

De este modo, lo que la lucha competitiva eterniza no son condiciones diferentes, sino las "diferencias de las condiciones" (Bourdieu, 2000: 164). Este tipo de lucha tiene sustento en el ejercicio de lo que Bourdieu denominó como "violencia simbólica"10. Esta violencia se ejerce cada vez que los dominados adhieren a los principios de legitimación de los dominantes.

La violencia simbólica se sustenta en el poder simbólico, como poder de constituir lo dado por la enunciación, de hacer ver $y$ de hacer creer, de confirmar o de transformar la visión del mundo y, de ese modo, la acción sobre el mundo, luego el mundo, que permite obtener el equivalente de lo que es obtenido por la fuerza física o económica, gracias al efecto específico de movilización, que no se ejerce si no es reconocido, es decir, desconocido como arbitrario (Bourdieu, 2006).

$10 \quad$ Este concepto fue formulado explícitamente en un primer momento para el ámbito escolar en $L a$ Reproducción (Bourdieu y Passeron, 1998). Para un recorrido histórico del concepto de violencia simbólica en la obra de Pierre Bourdieu, remitirse a Gutiérrez. "Poder, hábitus y representaciones: recorrido por el concepto de violencia simbólica en Pierre Bourdieu". Revista Complutense de Educación. 2004.
La "lucha revolucionaria", por otro lado, generaría una ruptura con el orden establecido. En esta, las clases dominadas se constituyen como un poder antagonista capaz de definir sus propios objetivos. Esta ruptura herética con el orden establecido, así como con las disposiciones $y$ representaciones que ese orden engendra entre los agentes modelados según sus estructuras, supone en sí misma una coincidencia entre el discurso crítico y una crisis objetiva, capaz de romper la concordancia inmediata entre las estructuras incorporadas y las estructuras objetivas de las que esas disposiciones $y$ representaciones son producto, e insistir en una especie de époche práctico, de suspensión temporal de la adhesión original al orden establecido (Bourdieu, 2001: 97).

La subversión herética, de este modo, explota la posibilidad de cambiar el mundo social cambiando la representación de ese mundo que contribuye a su realidad o, más concretamente, oponiendo una pre-visión paradójica, utopía, proyecto o programa a la visión ordinaria, que aprehende el mundo social como un mundo natural: enunciado preformativo, la pre-visión política es, en sí misma, una pre-dicción que pretende el acaecimiento de lo que enuncia (Bourdieu, 2001: 124). [De este modo], el discurso herético no sólo debe contribuir a romper la adhesión al mundo del sentido común profesando públicamente la ruptura con el orden ordinario, sino que debe también producir un nuevo sentido común e integrar en él, investidos con la legitimidad que confiere la manifestación pública y el reconocimiento colectivo, las prácticas y experiencias hasta ese momento tácitas o rechazadas por todo un grupo (Bourdieu, 2001: 97-98).

Es decir, Bourdieu distingue dos grandes modalidades de lucha: la revolucionaria, que pretende derrocar el orden establecido y en la que, las clases dominadas constituyen un poder antagonista capaz de definir sus propios objetivos $y$ por el contrario, la lucha competitiva, una especie de carrera en la cual los dominados 
se esfuerzan por apropiarse de las propiedades de los dominantes (Bourdieu, 2001: 103). Pero, cabe aclarar, que si bien conceptualmente Bourdieu establece esta distinción entre las modalidades que adquiere la lucha, se puede decir que la fuerza y el propósito principal de su obra fue dar cuenta empíricamente de los distintos modos en que se lleva a cabo la reproducción del orden social ${ }^{11}$. Así, en palabras de Bourdieu: "una de las preguntas más fundamentales a propósito del mundo social es la de saber por qué y cómo ese mundo perdura, persevera en el ser, cómo se perpetúa el orden social, es decir, el conjunto de las relaciones de orden que lo constituyen" (Bourdieu, 2007b: 31). Así, liga con ello a la sociología, la función de sacar a la luz las formas de dominación/dependencia que reproducen la vida social.

Para el sociólogo francés:

... el orden social, en lo esencial, produce su propia sociodicea. De modo que basta con dejar que actúen los mecanismos objetivos, o que actúen en nosotros, para otorgar al orden establecido, sin siquiera saberlo, su ratificación. Y quienes salen en defensa del orden simbólico amenazado por la crisis o la crítica, pueden limitarse a invocar las evidencias del sentido común, es decir, la visión de sí mismo que, salvo que ocurra una incidencia extraordinaria, el mundo social logra imponer. Podría decirse, haciendo un chiste fácil, que si el orden establecido está tan bien definido, es porque basta con un tonto para defenderlo (Bourdieu, 1999a: 239).

11 Sin embargo, analizar el orden social de ningún modo implica identificar a Bourdieu con los autores y perspectivas teóricas que, desde E. Durkheim $y$ pasando por los funcionalismos ( $y$ particularmente por el estructural-funcionalismo), buscan fundamentar el orden. En el marco de análisis críticos de la sociedad y sus relaciones, las preguntas acerca del orden, adquieren un sentido muy diferente. Para profundizar sobre este punto de la teoría de Bourdieu ver: Capdevielle, Julieta. "Dos teorías explicativas del orden social". Revista Question 21. 2009.
Para Wacquant, es así como:

la obra de Bourdieu puede leerse en su totalidad acertadamente como una búsqueda para explicar la especificidad y la potencia del poder simbólico, es decir, la capacidad que tienen los sistemas de sentido y significación para proteger $y$, por lo tanto, para reforzar, las relaciones de opresión y de explotación ocultándolas bajo el manto de la naturaleza, la benevolencia y la meritocracia (Wacquant 2005: 160 en Capdevielle, 2012).

Siguiendo este propósito, Bourdieu se ocupó de desentrañar a través de investigaciones empíricas las distintas modalidades que ha adquirido la lucha competitiva en la sociedad francesa.

En el próximo apartado, se centrará en una de las modalidades clave que adquiere la lucha de clases competitiva en la teoría bourdieussiana: las Estrategias de Reproducción Social. Este concepto se vuelve fundamental para entender como los agentes, especialmente, las familias o unidades domésticas ${ }^{12}$ luchan para mantener o mejorar su posición dentro del espacio social.

\subsection{ESTRATEGIAS DE REPRODUCCIÓN SOCIAL: UNA MODALIDAD DE LA LUCHA COMPETITIVA}

A través del concepto de Estrategias de Reproducción Social ${ }^{13}$, es posible entender las prácticas que las familias ponen en marcha para reproducirse socialmente, asi como su correlato y efecto en la dinámica de las clases sociales. Desde la perspectiva teórica

$12 \quad$ La unidad familiar o doméstica es un "grupo de personas que interactúan en forma cotidiana, regular $y$ permanente, a fin de asegurar mancomunadamente el logro de los siguientes objetivos: su reproducción biológica, la preservación de su vida, el cumplimiento de todas aquellas prácticas, económicas y no económicas, indispensables para la optimización de sus condiciones materiales y no materiales de existencia" (Gutiérrez, 2005: 45).

13 Para un listado exhaustivo de las distintas estrategias, ver Bourdieu, Pierre (1993). "Estrategias de reproducción y modos de dominación”. Campo del poder y reproducción social. Córdoba. Ferreyra Editor, 2007b: 31-49. 
de Pierre Bourdieu, las Estrategias de Reproducción Social se definen como:

... el conjunto de prácticas fenomenalmente muy diferentes, por medio de las cuales los individuos y las familias tienden, de manera consciente o inconsciente, a conservar o a aumentar su patrimonio, $y$ correlativamente a mantener o mejorar su posición en la estructura de las relaciones de clase (Bourdieu, 2000: 122).

Así, con este concepto, Bourdieu buscó integrar todas las prácticas que en situaciones $y$ contextos muy diferentes, agentes diferentes ponen en marcha y a través de las cuales se realiza la preservación de la unidad doméstica con el objetivo de reproducir su posición en el espacio social.

El origen del concepto de "estrategia" se sitúa alrededor de los comienzos de la década de 1960, a propósito de sus análisis sobre la sociedad Kabil y en el Béarn francés, ligados a la lógica de los intercambios matrimoniales y a las prácticas sucesorias: es allí cuando Bourdieu inicia su ruptura más profunda con el estructuralismo cuando pasa "de las reglas a las estrategias"14. Haciendo de esta última noción un concepto clave en su teoría de la acción, identificando a la misma con la noción de práctica y rescatando al agente social que produce las prácticas como resultado de las condiciones objetivas e incorporadas. El concepto de Estrategias de Reproducción Social es retomado luego como un aspecto central en el análisis de su obra fundamental: La Distinción (Gutiérrez, 2007a: 22-23).

La vinculación del concepto de Estrategias de Reproducción Social como una modalidad de la lucha competitiva, al abordar la dinámica de las clases y los mecanismos de perpetuación del orden social, nos provee de algunas ventajas analíticas en relación a otros conceptos ${ }^{15}$. En primer lugar, este concepto muestra claramente:

14 Para analizar este tema con mayor detenimiento, ver Bourdieu, Pierre. "De las reglas a las estrategias”. Cosas Dichas. 2007e: 67-82.

15 Para un análisis de este concepto y sus diferencias con otras tradiciones teóricas: Gutiérrez, Alicia B., 2005 .
... la concepción relacional de lo social, herencia estructuralista, que esta perspectiva teórica pone en juego. Así, las diferentes estrategias de reproducción social se explican sólo relacionalmente, en un doble sentido: en el contexto del sistema que constituyen (en una familia o en un grupo de familias pertenecientes a una clase o fracción de clase) y en el marco más amplio del espacio social global, donde las prácticas que forman parte de ese sistema se relacionan con las prácticas que son constitutivas de los otros, articulando de esa manera modos de reproducción diferenciales (Gutiérrez, 2007b: 20).

Por otro lado, hablar de "estrategias" implica otorgarle a la conducta de los actores una "autonomía relativa" respecto a la influencia de los factores macro sociales, razón por la cual estos actúan como "condicionantes" y no como "determinantes" (Gutiérrez, 2005: 46). Se sostiene así, la hipótesis de que existe una "lógica" en la configuración de los comportamientos familiares, que son susceptibles de variar de acuerdo a los parámetros impuestos por la pertenencia de clase (Gutiérrez, 2005:47). Las Estrategias de Reproducción varían, de este modo, según la composición del patrimonio que se trata de transmitir, es decir, el volumen $y$ el peso relativo de las diferentes especies de capital y por otra parte, el estado de los mecanismos de reproducción (Bourdieu, 2007b: 38).

El sistema de las estrategias de reproducción de una unidad doméstica depende de los beneficios diferenciales que puede esperar de las diferentes inversiones, en función de los poderes efectivos sobre los diferentes mecanismos institucionalizados (mercado económico, mercado escolar, mercado matrimonial) que le aseguran el volumen y la estructura de su capital (Gutiérrez, 2007: 20). [Especialmente], a través de la estructura de las posibilidades diferenciales de beneficio que son ofrecidas a sus inversiones por los diferentes mercados sociales, se imponen sistemas de preferencias (o de intereses) diferentes $y$ propensiones 
totalmente diferentes a invertir en los diferentes instrumentos de reproducción (Bourdieu, 2007b: 39).

A su vez, el uso del concepto de Estrategias de Reproducción implica tener en cuenta la "historicidad" del espacio social, de los distintos campos, pero también de los agentes y sus prácticas. Así, las Estrategias de Reproducción Social constituyen un proceso que se desarrolla a lo largo del ciclo vital familiar; las decisiones pasadas influyen en las presentes $y$ anticipan las futuras. Las decisiones relativas a cada una de las dimensiones conductuales de las estrategias familiares de vida están mutuamente interrelacionadas y por lo tanto, es necesario evitar crear “compartimentos estancos" (Gutiérrez, 2005: 47).

Por otra parte, resulta importante destacar que las Estrategias de Reproducción, "no tienen una intención consciente y racional", sino que las disposiciones del habitus tienden espontáneamente, a reproducir las condiciones de su producción. Debido al hecho de que estas dependen de las condiciones sociales de las que el habitus es producto, es decir, en las sociedades diferenciadas, del volumen y estructura del capital poseído por la familia (y de su evolución en el tiempo), las estrategias de las familias tienden a perpetuar su identidad, manteniendo distancias, relaciones de orden y contribuyendo así, en la reproducción de todo el sistema de las diferencias constitutivas del orden social (Bourdieu, 2007b: 37).

Por último, es importante mencionar que esta categoría incorpora la "dimensión simbólica". De hecho, la definición del modo de reproducción legítimo es una apuesta (enjuex) de luchas, especialmente en el seno del campo del poder económico (Bourdieu, 2007b: 47).

El concepto de Estrategias de Reproducción, con su foco en las unidades domésticas ${ }^{16}$, avanza hacia la articulación de las conductas individuales y las condiciones estructurales.

A continuación, se expondrán algunos ejemplos tomados de La distinción (2000), con el fin de ilustrar la puesta en marcha de las

16 Es claro que el "sujeto" de las Estrategias de Reproducción Social es, al menos en primer lugar, la "familia", considerada como "unidad doméstica".
Estrategias de Reproducción Social por parte de las familias, como una modalidad de la lucha competitiva. En relación al ámbito educativo, Bourdieu muestra cómo la competencia entre los grupos que luchan por mantener su posición dentro del espacio social, se organiza cada vez más en función de las titulaciones académicas.

La entrada en la carrera y en la competencia por la titulación académica de fracciones que hasta entonces han utilizado poco la escuela, ha tenido como efecto obligar a las fracciones de clase cuya reproducción estaba asegurada principalmente por la escuela, a intensificar sus inversiones para mantener la estructura de las clases, llegando a ser así la titulación académica y el sistema escolar que la otorga una de las apuestas privilegiadas de una competencia entre las clases que engendra un aumento general y continuo de la demanda de educación, y una inflación de las titulaciones académicas (Bourdieu, 2000: 130).

Frente a la permanente devaluación de las titulaciones, son múltiples las estrategias que los individuos $y$ las familias emplean para salvaguardar o mejorar su posición en el espacio social. Algunas de estas estrategias consisten en reconvertir sus especies de capital, hacer uso de su capital social ${ }^{17}$ para maximizar el rendimiento de las titulaciones obtenidas, intensificar sus inversiones en el campo educativo, así también, instituir límites de ingreso más selectivos, lo que constituye un numerus clausus, una especie de "proteccionismo consciente", que reclama a las instituciones que hagan al descubierto (con

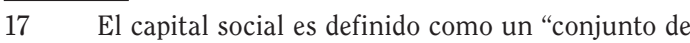
recursos actuales o potenciales que están ligados a la posesión de una red duradera de relaciones más o menos institucionalizadas de interconocimiento $y$ de inter-reconocimiento; o, en otros términos, a la pertenencia a un grupo, como conjunto de agentes que no están solamente dotados de propiedades comunes (susceptibles de ser percibidas por el observador, por los otros o por ellos mismos) sino que están también unidos por lazos permanentes y útiles" (Bourdieu, 1980: 2). 
límites netos y rígidos) lo que de manera invisible hacían unos mecanismos (de selección y segregación) que presentaban toda la apariencia de la necesidad natural (Bourdieu, 2000: 161).

La lucha por la apropiación de los bienes económicos o culturales - parte constitutiva de las estrategias de reproducción social- es inseparable de su dimensión simbólica. De este modo, los bienes constituyen signos distintivos enclasantes y con ello, dadores de identidad. La cultura es una apuesta que, como todas las apuestas sociales, supone e impone a la vez que se entre en el juego y que se tome gusto al juego; asimismo, el interés por la cultura, sin el que no existe carrera, concurso, competencia, se produce por las mismas carreras y competencia que produce (Bourdieu, 2000: 247).

En síntesis, las luchas sociales —en su modalidad competitiva- son siempre luchas por la apropiación de bienes, pero también por la imposición de las maneras de ver el mundo social; son luchas de clasificación y a la vez, luchas de definición de clases. Develando los mecanismos de esta doble lucha, la ciencia social da los medios para contribuir a la producción de las transformaciones de la sociedad (Martínez, 2007: 236).

\section{CONCLUSIONES: ¿LUCHA Y CAMBIO SOCIAL?}

"Así, paradójicamente, la sociología libera al liberar de la ilusión de la libertad, o, más exactamente, de la creencia mal ubicada en las libertades ilusorias. La libertad no es algo dado, sino una conquista, y colectiva" (Bourdieu, 2007a).

Tal como se ha analizado a lo largo de este artículo, desde la perspectiva bourdieussiana, las luchas sociales no están todas ni siempre en contradicción con la perpetuación del orden establecido. Más allá de las antítesis, la permanencia puede estar asegurada por el cambio y la estructura perpetuada por el movimiento (Bourdieu, 2000). Muchos trabajos sobre la obra de Pierre Bourdieu destacan el aporte realizado por este autor a la comprensión y explicación de los mecanismos, a través de los cuales se realiza la reproducción de la desigualdad social, gracias a su explicitación en diversas investigaciones empíricas. Sin embargo, se ha querido aportar haciendo hincapié en el análisis de las dos formas que supone la lucha de clases desde la mirada analítica bourdieussiana: la lucha competitiva y la lucha revolucionaria.

Para ello, a partir de la indagación en algunas de sus investigaciones empíricas y centrándose en el concepto de Estrategias de Reproducción Social, se han presentado algunos ejemplos acerca de los diversos planos en los que se despliega la lucha competitiva. Particularmente, la competencia por la acumulación y apropiación de títulos escolares, así como las prácticas de distinción en el campo cultural.

Asimismo, el concepto de lucha competitiva resulta particularmente iluminador, puesto que, por un lado permite comprender el modo en que se desarrolla la lucha de clases en los múltiples planos que abarcan el conjunto de las prácticas sociales de la vida cotidiana y por otro, al hacer hincapié en este mecanismo social, comprender al conflicto como un elemento inherente y constitutivo de lo social.

Por otra parte, se ha pretendido destacar el peso que Bourdieu le otorga a la dimensión simbólica en el concepto de lucha de clases. Así, es necesario recordar que, si de la obra de Marx, Bourdieu ha tomado que la realidad social es un conjunto de "relaciones de fuerza entre clases históricamente en lucha unas con otras", por otro lado de la obra de Weber, ha tomado que la realidad social es también un conjunto de "relaciones de sentido" y que toda dominación social, a menos de recurrir pura y continuamente a la violencia armada, debe ser reconocida y aceptada como legítima, es decir, tomar un sentido, preferentemente positivo, de manera que los dominados adhieran al principio de su propia dominación y se sientan solidarios de los dominantes en un mismo consenso sobre el orden establecido (Gutiérrez, 2004: 292). Este mecanismo social está implicado en el concepto de violencia simbólica.

Sin embargo, a diferencia de Marx, para Bourdieu la lucha de clases es una lucha que, por medio de su propia eficacia y según la modalidad de la misma, puede ayudar a perpetuar o modificar el orden social. Lucha competitiva o revolucionaria respectivamente. 
Se ha argumentado también, que la lucha por las clasificaciones forma parte de la lucha de clases. La representación del mundo social no es un dato o lo que viene a ser lo mismo, un registro, un reflejo; por el contrario, es un acto de construcción desigualmente distribuido en las diferentes posiciones del espacio social. Así, resulta necesario reconstruir la génesis social de los conceptos, clasificaciones y categorías de percepción como productos históricos de luchas históricas que la amnesia de la génesis eterniza y reífica.

Teniendo en cuenta lo anterior, Bourdieu plantea que la sociología, como disciplina que intenta comprender y explicar el mundo social, adquiere un papel importante en las disputas de clase. Para este autor, resulta fundamental un ejercicio crítico de la sociología que reconstruya las condiciones sociales de producción de las categorías sociales de percepción y de representación del mundo natural o social. Es precisamente gracias a esta naturaleza simbólica de la lucha por la construcción legítima del mundo, que la sociología se constituye como una ciencia política, disciplina central en el campo de las luchas simbólicas por la transformación de la sociedad.

Sin embargo, "en la lucha por la imposición de la visión legítima, en que la propia ciencia está inevitablemente empeñada, los agentes detentan un poder proporcional a su capital simbólico, es decir, al reconocimiento que reciben de un grupo" (Bourdieu, 2001: 66). Así, la violencia simbólica implícita en todo orden simbólico se relaciona con el poder de nominación desigualmente distribuido entre los diferentes grupos sociales, a partir de la disposición y apropiación de un volumen diferencial de capital simbólico. Por este motivo, dominadas hasta en la producción de su imagen del mundo social y en consecuencia de su identidad social, las clases dominadas no hablan, sino que son habladas (Bourdieu, 2007c: 175), mientras que los dominantes imponen las normas de su propia objetivación, reduciendo su verdad objetiva a su intención subjetiva.

Quienes ocupan las posiciones dominadas en el espacio social, también están situados en posiciones dominadas en el campo de la producción simbólica y no poseen los instrumentos de producción necesarios para expresar su propio punto de vista acerca de lo social. De este modo, en la lucha simbólica por la producción de un sentido común antagónico a los intereses de los dominantes, en la lucha revolucionaria para la transformación social, las armas están desigualmente distribuidas.

El mundo social es producto de lo que hacen los agentes a cada momento, pero solo pueden deshacerlo o rehacerlo sobre la base de un conocimiento realista de lo que este mundo es $y$ de lo que ellos pueden hacer en función de la posición que en este ocupan (Bourdieu, 1990: 298). Así, el intelectual o sociólogo, como portador de capital simbólico, susceptible de ser utilizado en el campo de la acción política, utilizando las armas que posee y domina, a través del develamiento de las relaciones de dominación, está en condiciones de ofrecer a los dominados, sobre la base de una homología de posición, los instrumentos de ruptura con las representaciones que se engendran en la complicidad inmediata de las estructuras sociales $y$ mentales que tienden a asegurar la reproducción continuada del orden.

A los ojos de Bourdieu, el trabajo sociológico es desnaturalizar $y$ desfatalizar el mundo social, esto es, destruir los mitos que ocultan el ejercicio del poder y la perpetuación de la dominación. De este modo, la sociología de Bourdieu también puede leerse como una política en el sentido que él da al término, un intento por transformar los principios de la visión, mediante los cuales se construye (y por lo tanto, se puede formar racional $y$ humanamente) la sociología, la sociedad y en última instancia, a nosotros mismos.

\section{BIBLIOGRAFÍA}

Bourdieu, Pierre. "Le capital social. Notes provisoires". Revista Actes de la Recherche en Sciences Sociales 31. 1980: 2-3.

Bourdieu, Pierre. "Espacio social y génesis de las clases”. Sociología y Cultura. México. Editorial Grijalbo, 1990: 281-310.

Bourdieu, Pierre. Meditaciones Pascalianas. España: Editorial Anagrama, 1999a. 
Bourdieu, Pierre. "La economía de los bienes simbólicos". Razones prácticas. Sobre la teoría de la acción. Barcelona. Editorial Anagrama, 1999b: 159-198.

Bourdieu, Pierre. La distinción, criterios y bases sociales del gusto. España: Editorial Taurus, 2000.

Bourdieu, Pierre. ¿Qué significa hablar? Economía de los intercambios lingüísticos. España: Editorial Akal, 2001.

Bourdieu, Pierre (1999). "Sobre el poder simbólico". Intelectuales, política y poder. Argentina. Editorial EUDEBA, 2006: 65-73.

Bourdieu, Pierre (1987). "Espacio social y poder simbólico". Cosas dichas. España. Editorial Gedisa, 2007a: 127-142.

Bourdieu, Pierre (1993). "Estrategias de reproducción y modos de dominación". Campo del poder y reproducción social. Argentina. Editorial Ferreyra, 2007b: 31-49.

Bourdieu, Pierre. "Una clase objeto". Campo del poder y reproducción social. Argentina. Editorial Ferreyra, 2007c: 171-178.

Bourdieu, Pierre. La miseria del mundo. Argentina: Editorial Fondo de Cultura Económica, 2007d.

Bourdieu, Pierre. "De las reglas a las estrategias". Cosas dichas. Buenos Aires. Gedisa, 2007e: 67-82.

Bourdieu, Pierre y Passeron, Jean Claude. La reproducción. Elementos para una teoría del sistema de enseñanza. México: Editorial Fontamara, 1998.

Bourdieu, Pierre y Wacquant, Loic. Una invitación a la sociología reflexiva. Buenos Aires: Siglo XXI, 2008.

Bourdieu, Pierre; Chamboredon, Jean Claude y Passeron, Jean Claude. El oficio del sociólogo: presupuestos epistemológicos. Argentina: Editorial Siglo XXI, 2008.

Capdevielle, Julieta. "Dos teorías explicativas del orden social". Revista Question 21. Argentina: Facultad de Periodismo y Comunicación Social, Universidad Nacional de La Plata, 2009.

Capdevielle, Julieta. "La sociología figuracional de Norbert Elías y el estructuralismo genético de Pierre Bourdieu: encuentros y desencuentros". Aposta. Revista de Ciencias Sociales 52. España, 2012.

Costa, Ricardo. "Entre la necesidad y la libertad: condiciones sociales del cambio en Pierre Bourdieu". Revista Estudios Sociológicos xxIV (001). México. El Colegio de México-Distrito Federal, enero-abril 2006: 167-196.

Cragnolino, Elisa. "La noción de espacio social rural en el análisis de procesos de acceso a la educación de jóvenes y adultos $y$ apropiación de cultura escrita". Procesos de alfabetización y acceso a la educación básica de jóvenes y adultos. María Lorenzatti (comp.). Argentina. Editorial Vaca Narvaja, 2011: 191-209.

Gutiérrez, Alicia. "Poder, hábitus y representaciones: recorrido por el concepto de violencia simbólica en Pierre Bourdieu". Revista Complutense de Educación 15 (1). 2004: 289-300.

Gutiérrez, Alicia. Pobre: como siempre... Estrategias de reproducción social en la pobreza. Argentina: Editorial Ferreyra, 2005.

Gutiérrez, Alicia. "Clases, espacio social y estrategias: una introducción al análisis de la reproducción social en Bourdieu". Campo del poder y reproducción social. Pierre Bourdieu. 2007a: 9-27.

Gutiérrez, Alicia. "Herramientas teóricometodológicas de un análisis relacional para los estudios de la pobreza". Revista Ciencia, Docencia y Tecnología 35. Concepción del Uruguay, 2007b: 15-33.

Martínez, Ana Teresa. Pierre Bourdieu. Razones y lecciones de una práctica sociológica. Argentina: Editorial Manantial, 2007.

Wacquant, Loic. "Tras las huellas del poder simbólico. La disección de la "nobleza de Estado". El misterio del ministerio. Pierre Bourdieu y la politica democrática. Loic Wacquant (comp.). España: Editorial Gedisa, 2005.

Fecha de ingreso: $01 / 08 / 2012$ Fecha de aprobación: 07/11/2012 